SCIENCE CHINA

Physics, Mechanics \& Astronomy

\title{
Experimental test of robustness of composite pulses to non-static classic noise in NMR
}

\author{
XinYu Pan ${ }^{*}$ \\ Institute of Physics, Chinese Academy of Sciences, Beijing 100190, China
}

Received October 18, 2016; accepted October 26, 2016

Citation: X. Y. Pan, Experimental test of robustness of composite pulses to non-static classic noise in NMR, Sci. China-Phys. Mech. Astron. 60, 020333 (2017), doi: $10.1007 /$ s11433-016-0412-6

High fidelity and robust quantum control is the requirement for Quantum Information Processing (QIP) and quantum computing applications. Many negative factors, like pulse length error, system-bath coupling and the fluctuation of electronic instruments, deviate the quantum system evolution from the ideal path. To suppress these errors, many quantum manipulation techniques have been proposed. Composite pulses are designed to suppress off resonance error, pulse length error or the combination of them. The optimization of composite pulses is based on the quasi-static assumption, where the noise is constant in every single experiment. However, in realistic situation, the bath evolving and the electronic instrument fluctuation will make these errors time-varying. If the fluctuation period is comparable with the control time, the quasi-static assumption will fail.

In ref. [1], the performance of composite pulses under time-varying hybrid errors is analyzed under filter function formalism. The investigation shows the advantage of composite pulses (CP) over primitive pulses in low-frequency noise region and the failure of CPs in high-frequency noise
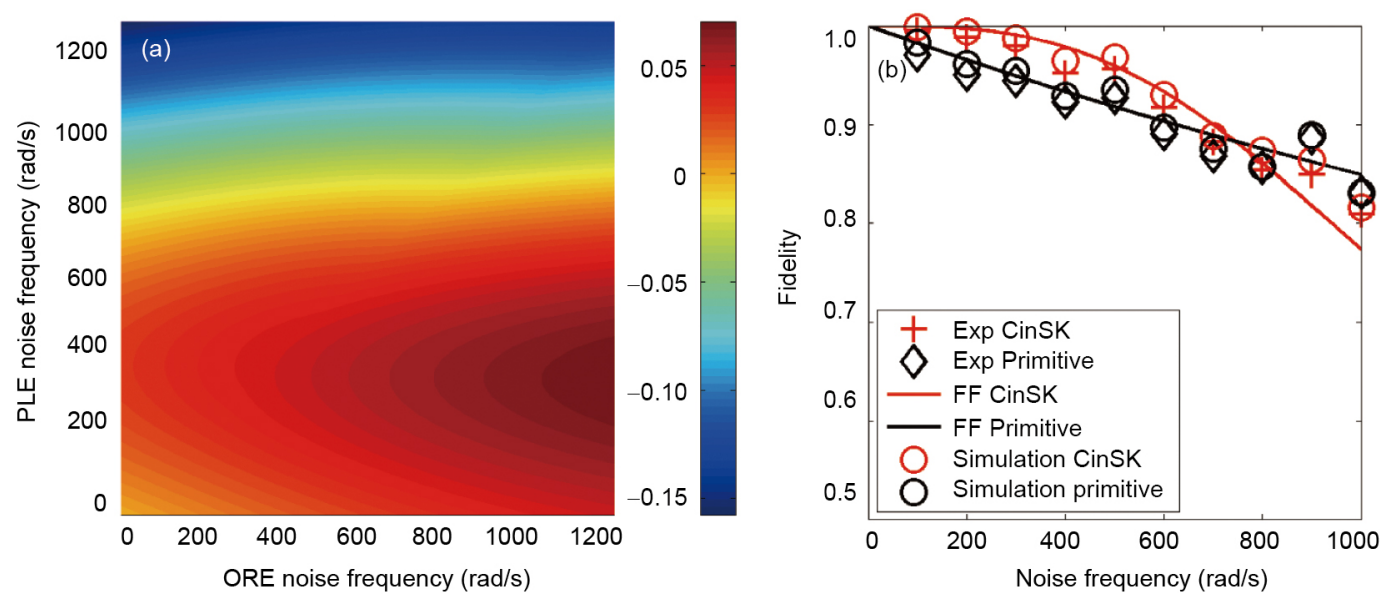

Figure 1 (Color online) (a) Evolution fidelity difference between reduced CinSK and primitive $\pi$ pulse. (b) Comparison of experimental data (cross and diamond markers) with FF prediction (solid lines) and Numerical Simulation results (circles). The rabi frequency is also set as $3125 \pi$. The noise PSD is $S(\omega) \sim C$, where $C$ is a constant number, and the noise amplitude for the two errors are satisfying $\alpha_{\Omega} \equiv \alpha_{f}=100[1]$.

*Corresponding author (email: xypan@iphy.ac.cn) 
region (Figure 1). The hybrid noise can be effectively simulated by modulating the control pulse shapes. The experiment in NMR system validated the prediction for CPs robustness and gave the noise cut frequency threshold [1].

The robustness of CPs in low-frequency region guarantee CPs can work in more realistic situation. The analysis in filter function formalism offered us a method to find practical CPs according to experimental noise properties. The suppression capacity of reduced CinSK composite pulses to the pulse length error is stronger than the capacity to the off resonance error. Simulation results indicate that the evolution of concatenated composite pulses is sensitive to the qubit initial states.

1 X. L. Zhen, T. Xin, F. H. Zhang, and G. L. Long, Sci. China-Phys. Mech. Astron. 59, 690312 (2016) 